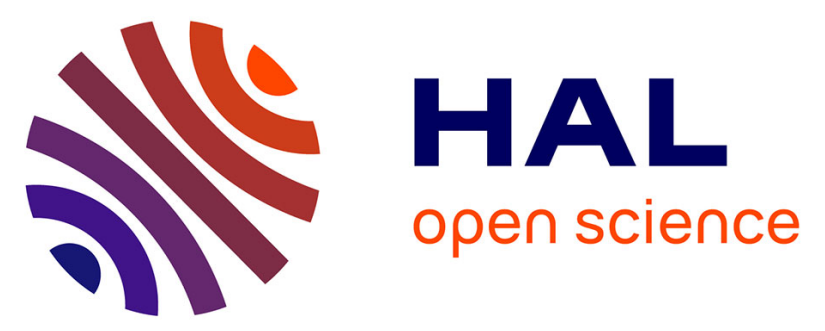

\title{
A pilot study of dual isotope lymphoscintigraphy for breast sentinel node biopsy comparing intradermal and intraparenchymal injection
}

J Charlotte Fowler, Chandra K Solanki, Ilonka Guenther, Robert Barber, Fiona Miller, Lynda Bobrow, Duraisamy Ravichandran, David Lawrence, James R Ballinger, Anthony Douglas-Jones, et al.

\section{To cite this version:}

J Charlotte Fowler, Chandra K Solanki, Ilonka Guenther, Robert Barber, Fiona Miller, et al.. A pilot study of dual isotope lymphoscintigraphy for breast sentinel node biopsy comparing intradermal and intraparenchymal injection. EJSO - European Journal of Surgical Oncology, 2009, 35 (10), pp.1041. 10.1016/j.ejso.2009.02.018 . hal-00556289

\section{HAL Id: hal-00556289 https://hal.science/hal-00556289}

Submitted on 16 Jan 2011

HAL is a multi-disciplinary open access archive for the deposit and dissemination of scientific research documents, whether they are published or not. The documents may come from teaching and research institutions in France or abroad, or from public or private research centers.
L'archive ouverte pluridisciplinaire $\mathbf{H A L}$, est destinée au dépôt et à la diffusion de documents scientifiques de niveau recherche, publiés ou non, émanant des établissements d'enseignement et de recherche français ou étrangers, des laboratoires publics ou privés. 


\section{Accepted Manuscript}

Title: A pilot study of dual isotope lymphoscintigraphy for breast sentinel node biopsy comparing intradermal and intraparenchymal injection

Authors: J Charlotte Fowler, Chandra K Solanki, llonka Guenther, Robert Barber, Fiona Miller, Lynda Bobrow, Duraisamy

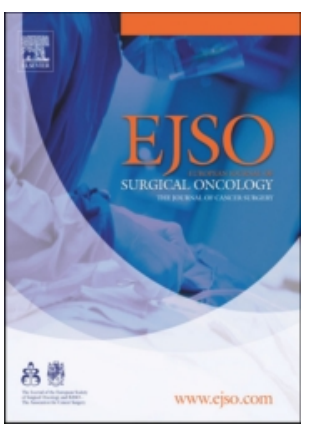

Ravichandran, David Lawrence, James R Ballinger, Anthony

Douglas-Jones, Arnie D Purushotham, A Michael Peters

PII: $\quad$ S0748-7983(09)00075-4

DOI: $\quad$ 10.1016/j.ejso.2009.02.018

Reference: $\quad$ YEJSO 2805

To appear in: European Journal of Surgical Oncology

Received Date: 23 January 2008

Revised Date: 23 February 2009

Accepted Date: 24 February 2009

Please cite this article as: Fowler JC, Solanki CK, Guenther I, Barber R, Miller F, Bobrow L, Ravichandran D, Lawrence D, Ballinger JR, Douglas-Jones A,, Purushotham AD, Peters AM. A pilot study of dual isotope lymphoscintigraphy for breast sentinel node biopsy comparing intradermal and intraparenchymal injection, European Journal of Surgical Oncology (2009), doi: 10.1016/j.ejso.2009.02.018

This is a PDF file of an unedited manuscript that has been accepted for publication. As a service to our customers we are providing this early version of the manuscript. The manuscript will undergo copyediting, typesetting, and review of the resulting proof before it is published in its final form. Please note that during the production process errors may be discovered which could affect the content, and all legal disclaimers that apply to the journal pertain. 


\title{
A pilot study of dual isotope lymphoscintigraphy for breast sentinel node biopsy comparing intradermal and intraparenchymal injection
}

\author{
${ }^{1,2,5} \mathbf{J}$ Charlotte Fowler \\ ${ }^{1}$ Chandra K Solanki \\ ${ }^{1}$ Ilonka Guenther \\ ${ }^{1}$ Robert Barber \\ ${ }^{2}$ Fiona Miller \\ ${ }^{3}$ Lynda Bobrow \\ ${ }^{6}$ Duraisamy Ravichandran \\ ${ }^{7}$ David Lawrence \\ 1, a James R Ballinger \\ ${ }^{8}$ Anthony Douglas-Jones \\ ${ }^{4, a}$ Arnie D Purushotham \\ ${ }^{1, \mathrm{~b}}$ A Michael Peters
}

Departments of ${ }^{1}$ Nuclear Medicine, ${ }^{2}$ Radiology and ${ }^{3}$ Histopathology, and ${ }^{4}$ Cambridge Breast Unit, Addenbrooke's Hospital, Cambridge, Departments of ${ }^{5}$ Radiology, ${ }^{6}$ Surgery and ${ }^{7}$ Pathology, Luton and Dunstable Hospital, Luton, ${ }^{8}$ Department of Histopathology, University of Wales College of Medicine. Current affiliations: ${ }^{\mathrm{a}}$ King's College London/Guy's and St Thomas' NHS Foundation Trust and ${ }^{\mathrm{b}}$ Brighton Sussex Medical School.

Author for correspondence:

Dr Charlotte Fowler

email: charlotte.fowler@ldh.nhs.uk

Luton and Dunstable NHS Trust

Lewsey Road

Luton

LU4 0DZ

Short title: Dual isotope dual level breast lympho-scintigraphy

Financial Support: Royal College of Radiologists of the United Kingdom 


\section{Abstract}

AIMS: Identification of sentinel lymph nodes (SLN) may depend on the tissue plane of tracer injection. To explore this, we developed a dual isotope technique to compare the lymphatic drainage basins accessed by intradermal and parenchymal injections.

METHODS: Fifteen breast cancer patients had simultaneous parenchymal and intradermal injections of ${ }^{99 \mathrm{~m}}$ Tc-labelled human immunoglobulin G (HIG) and ${ }^{111}$ In-HIG, respectively, 2-4 h before axillary lymph node clearance surgery. All 228 freshly dissected nodes were assayed by well-counting and examined for metastatic disease by haematoxylin/eosin staining and immunohistochemistry.

RESULTS: Total nodal uptake following intradermal injection was 10 times more than after parenchymal injection. Tracer uptake within the first three draining nodes divided patients into 3 groups; 4(group1) had identical $1^{\text {st }}, 2^{\text {nd }}$ and $3^{\text {rd }}$ echelon nodes, 6(group2) had identical $1^{\text {st }}$ and $2^{\text {nd }}$ echelon nodes and 5(group3) had different $1^{\text {st }}$ echelon nodes. With respect to the first, second and third groups, there was close, moderate and poor correlation (Pearson), respectively, between individual nodal counts accumulated from the two injection sites. Of 8 patients with nodal disease, the SLN identified by intradermal and parenchymal injections contained disease in 7 and 4 patients, respectively.

CONCLUSIONS: Comparison of nodal tracer distributions from the two injection planes allows a functional model to be developed with two possible routes of drainage from the parenchymal plane, one joining the tract from the aereolar plexus and the other passing independently to the axilla which builds upon Sappey's original anatomical model. This may explain the variable uptake, discordance and false negative SLN identification. 


\section{Introduction}

Sentinel lymph node (SLN) localisation is an elegant technique that aims to reduce unnecessary lymph node clearance in patients undergoing surgery for breast cancer and other neoplasms that spread via lymphatics. Based on the concept of lymphatic spread in a systematic fashion from the tumour to the SLN, and then on to the remainder of the draining lymphatic basin, it relies on the use of a tracer that faithfully reproduces the route taken by metastatic cells.

SLN localisation in the staging of breast cancer has been enthusiastically adopted to minimise potentially debilitating surgery in the $70-80 \%$ of subjects who have early disease (T1-2N0M0) and a healthy axilla [1]. However, unlike the clinical setting of melanoma, in which it has been shown that when the SLN is clear of disease there are few false negative 'skip lesions' (ie metastatic disease further 'down stream' in the presence of a clear SLN) [2,3], the false negative rate in breast cancer may be higher, reported up to $22 \%$ in some centres [4]. Such false negative SLN subjects will be under-staged and receive inappropriate treatment that may only become apparent after many years, given the prolonged natural history of breast cancer.

Breast lymphatics are traditionally thought to comprise two interconnecting systems; a deep system that tracks the blood vessels around the alveoli, and the parenchyma of the breast, and a superficial system that follows the lactiferous system to the nipple and skin. It is uncertain which of these two routes is favoured by cells metastasising to the axilla [5-15]. Only when this has been established will the evidence be in place to guide the ideal injection site for axillary SLN identification. 
Three general approaches have been used in previous studies to address this problem. Firstly, comparison between two injection sites in different subjects using the same tracer [5-10]; secondly, comparison between different tracers (usually blue dye and a particulate radioisotope tracer) injected simultaneously in different planes within the same subject [10-13]; and thirdly, comparison between different tissue plane injections at different times in the same subject using the same radiotracer $[14,15]$. These studies have had mixed outcomes, some finding that injections into different tissue planes give a discordant nodal distribution [6-8,14] whereas others, a concordant pattern [5,9-11,13]. This may be secondary to poorly matched subjects in the first approach, differently behaving tracers in terms of molecular dimensions and different lymphatic tracer handling in the second, and day-to-day variations in patient physiology in the third. All have relied on gamma-camera imaging and/or intra-operative probe monitoring which, in the context of determining differential routes of drainage, have limited functional and anatomical resolution. Fuelled by a paucity of prospective randomised trials to establish the optimal technique [16], the controversy regarding the ideal tissue plane for injection remains unresolved.

The aim of the current study was to develop a different strategy to clarify the position, based on the ex vivo radioassay of all nodes removed during level 2 axillary clearance, to determine the extent to which the axillary lymphatic drainage basins respectively accessed by intradermal and parenchymal tracer injections are similar to each other, and in the event of significant differences, the extent to which each reflects the route taken by metastasising cancer cells, with a view ultimately to determining the ideal injection site or combination of them to identify the 
SLN. We elected to use a single agent, dual labelled with different separately detectable isotopes administered simultaneously into the intradermal and parenchymal planes. Only tracers proven to have near-identical functional behaviour in the lymphatic system would be acceptable, otherwise inherent differences in behaviour may be misinterpreted as differences in lymphatic flow.

Micro-aggregated human serum albumin (nanocolloid [NC]) is the most commonly used lymphoscintigraphic agent, at least in Europe [17]. Although some workers have reported success in dual isotope labelling of NC with ${ }^{99 \mathrm{~m}} \mathrm{Tc}$ and ${ }^{111}$ In [18], at least on in vitro testing, our attempts to achieve stable dual labelling were unsuccessful as judged by discordant in vivo data, despite favourable in vitro results [19]. We therefore used human polyclonal immunoglobulin G (HIG), a soluble macromolecule with a size (150 kDa) that favours uptake into lymphatics rather than blood capillaries. It is a useful lymphoscintigraphic agent [20,21] that has recently been shown to identify the same sentinel and $2^{\text {nd }}$ echelon nodes as ${ }^{99 m}$ Tc-NC [22]. Moreover, it has been shown that ${ }^{99 \mathrm{~m}} \mathrm{Tc}-\mathrm{HIG}$ and ${ }^{111} \mathrm{In}$-HIG display almost identical tissue-to-blood transport kinetics [23, 24].

\section{Materials and methods}

\section{Subjects}

Fifteen subjects were recruited, all female aged 45 - 81, with biopsy-proven early breast cancer, stage T1-2N0M0, scheduled to have excision of their primary tumour and level 2 axillary clearance. All gave written informed consent. The study was approved by the Local Research 
Ethics Committees of both study centres (Cambridge and Luton) and by the Administration of Radioactive Substances Advisory Committee of the United Kingdom.

\section{Radiopharmaceuticals}

${ }^{111}$ In-HIG was prepared by mixing $5 \mathrm{MBq}{ }^{111}$ In-chloride with $1 \mathrm{~N}$ sodium acetate and adding 1 mg HIG (Sandoglobulin, Novartis Pharmaceuticals UK Ltd, Frimley, Surrey) which had been derivatised with the bifunctional chelator, p-isothiocyanatobenzyl DTPA. The product was then diluted with human immunoglobulin ( $5 \mathrm{mg} / \mathrm{ml}$ in $0.1 \mathrm{~N}$ bicarbonate) to neutralise the $\mathrm{pH}$. Radiochemical purity, measured by thin-layer chromatography, was $93.4 \pm 3.2 \%$.

${ }^{99 \mathrm{~m}}$ Tc-HIG was prepared by addition of approximately $400 \mathrm{MBq}$ sodium $\left[{ }^{99 \mathrm{~m}} \mathrm{Tc}\right]$ pertechnetate, eluted from a generator with less than $24 \mathrm{~h}$ in-growth, to a commercial kit (Technescan HIG, Tyco Healthcare, Gosport, Hampshire). Radiochemical purity, measured by thin layer chromatography, was $98.5 \pm 1.1 \%$.

${ }^{99 \mathrm{~m}}$ Tc-HIG (10 MBq) and ${ }^{111} \mathrm{In}-\mathrm{HIG}(2 \mathrm{MBq})$ were both administered in volumes of $0.2 \mathrm{ml}$. The administered activities were chosen to minimise cross-talk between the two radionuclides, taking account of previous pilot studies that showed significantly higher activities entering lymph nodes following intradermal injection compared with parenchymal injection.

\section{Procedure}

The radiotracers were injected 2-4 h before resection of the primary lesion and level 2 axillary clearance. ${ }^{99 \mathrm{~m}}$ Tc-HIG was injected into the peri-tumoural parenchymal plane under ultrasound 
guidance and ${ }^{111}$ In-HIG injected intradermally at the areola. True intradermal placement was confirmed by the elevation of a wheal at the injection site.

Immediately following surgery, the axillary specimen was freshly dissected using palpation and scalpel to separate individual lymph nodes. Each node was placed into a $20 \mathrm{ml}$ glass countingbottle and filled with phosphate-buffered formalin to a volume of $4 \mathrm{ml}$. Diluted standards containing known fractions of the administered activity were prepared, along with water standards for background counts. Lymph node activity was assayed using a well-counter (Wallac 1480 Wizard automatic gamma counter [Wallac, Turku, Finland] or Scaler Ratemeter SR7 connected to a 2” sodium iodide gamma well counter [Nuclear Enterprises Ltd, Reading, UK]) using windows chosen to minimise cross-talk between the 2 radionuclides. The counts in each node were corrected for dead-time, background, cross-talk and radionuclide physical decay, and expressed as a percentage of the administered activity.

\section{Histopathology}

All nodes were left to fix overnight in phosphate-buffered formalin. Nodes smaller than $5 \mathrm{~mm}$ in maximum diameter were processed and embedded whole. Nodes greater than $5 \mathrm{~mm}$ were sliced at approximately $5 \mathrm{~mm}$ intervals. All were embedded in paraffin wax. Four $\mu \mathrm{m}$ sections were cut at one level and stained in an automatic stainer with haematoxylin and eosin (H\&E). The specimens were reported as negative or positive for metastatic disease according to national reporting guidelines [25], and patients managed accordingly. All nodes were subjected to further analysis by immuno-histochemistry (IHC) using high and low molecular weight anti-cytokeratin antibodies, AE1/AE3, on sections at 2 levels, $40 \mu \mathrm{m}$ apart, as recommended by Turner et al [26]. 
The areas of the nodes and of the metastatic deposits within them were measured in $\mathrm{mm}^{2}$ from stained sections to semi-quantify nodal sizes and disease burden within individual involved nodes.

\section{Statistics}

Patients were sub-divided into 3 groups according to concordance between intradermal and intraparenchymal injection sites with respect to identification of the three 'hottest' nodes (see Results). Concordance between nodal lymphatic tracer contents was quantified using Pearson’s correlation coefficient $\left(\mathrm{R}^{2}\right)$ and compared between the 3 groups. The ratio of total counts accumulated in the resected specimens was compared with unity using Student's t-test.

Results Of the 15 patients, 8 had ductal and 4 had lobular carcinoma, while in 3 it was mixed. The median number of nodes dissected was 15 (range 5 - 19).

Lymph node activity accumulated from deep and superficial planes

In one subject $(\mathrm{J})$, the axillary nodes accumulated $15 \%$ of activity administered by intradermal injection, presumably as a result of inadvertent direct cannulation of a lymphatic vessel.

Otherwise, the resected nodes accumulated no more than $1 \%$ of administered activity. There was more accumulation of nodal tracer uptake following intradermal injection compared with intraparenchymal injection, with a mean ratio of 10.2 (SD 10.3; $<<0.01$ versus unity, excluding patient J).

Patterns of lymphatic tracer distribution 
In 4 patients, the two injection sites identified the same $1^{\text {st }}, 2^{\text {nd }}$ and $3^{\text {rd }}$ echelon nodes (group 1 ). In 6 patients, the $1^{\text {st }}$ and $2^{\text {nd }}$ nodes were the same but not the $3^{\text {rd }}$ (group 2) and in 5 patients, the injection sites identified different $1^{\text {st }}$ echelon nodes (group 3). Correlation between the injections sites with respect to individual nodal activity was very high in group $1\left(\mathrm{R}^{2}=0.99\right.$ in all 4$)$, moderately high in group 2 (median $\mathrm{R}^{2}=0.9$; range $0.49-0.93$ ) but generally poor in group 3 (median $\mathrm{R}^{2}=0.16$; range $0.01-0.94$ ). There were no significant differences across the groups with respect to tumour grade, size, or location, or the ratio of total nodal activities accumulated from the respective injection sites.

Nodal disease versus tracer uptake from the two planesIn 7 patients, the axilla was free of disease. A total of 16 nodes harboured disease in the remaining 8 patients. The 'hottest'node contained disease in 7/8 patients after intradermal injection and in 4/8 patients after parenchymal injection. The single false negative SLN identified by intradermal injection (patient G) ranked $11^{\text {th }}$ in isotope content, compared with $2^{\text {nd }}$ after parenchymal injection. This patient was the only subject to have received tracer injections at the same time as wire localisation. With her exclusion from the analysis and use of IHC to identify micrometastatic disease, the sensitivity for intradermal injection was therefore $100 \%$, compared with $50 \%$ for parenchymal injection.

In 3 of the 4 patients who had more than one diseased node, there was an inverse trend between rank based on isotope content and rank based on disease load.

The largest node in the resected specimen contained disease in only 3/8 patients. When nodes were ranked in terms of size, the average rank of nodes containing metastatic disease was $6^{\text {th }}$. 
IHC identified nodal disease not diagnosed by H\&E staining in 2/8 patients. In one of these (L), the SLN from both routes was the only node to contain disease and use of IHC upgraded the patient from N0 to N1. In the other (K), the SLN from the intradermal route contained a micrometastasis identifiable only by IHC, even though a macro-metastasis was identified in a lower order node ( $4^{\text {th }}$ echelon from both the intradermal and parenchymal routes).

\section{Discussion}

Justification of the study

The most active lymph node resulting from administration of a radiotracer corresponds to the SLN only if the radiotracer is taken up into and transported through the same lymphatics as the potential metastatic cell. The radiotracer acts as a surrogate for cancer cells, providing a road map for surgical selection, thereby making an appropriate site of tracer injection crucial for the reliability of the technique.

Previous lymphoscintigraphic imaging studies found that parenchymal injection demonstrated lymphatic flow to the internal mammary chain as well as the axilla in $11-14 \%$ of patients, in contrast to intradermal injection which showed nodal uptake only in the axilla [14]. There is no doubt that the parenchymal injection outperforms intradermal injection for demonstrating global lymphatic distribution from the whole breast, which includes the internal mammary chain. Lymphatic staging in breast carcinoma is used to direct surgical management of the axilla, , so which of these two routes best stages the axilla is the main practical issue. As no consensus has 
yet been reached [16], our study was undertaken as a pilot study, to evaluate a novel approach involving detailed examination of two drainage basins in relation to the routes taken by cancer cells metastasising to the axilla. The dual-isotope technique described here, based on a single agent (HIG), was developed specifically to allow separate quantification of two lymphatic radiotracers following concurrent injection into two different planes. Well-counting of all individual nodes gave absolute confidence in correlating nodal isotope content with pathology on a node-by-node basis. Such accurate dual window radiotracer quantification of all axillary nodes is not possible either with intra-operative, hand-held gamma probe monitoring or gamma camera imaging because of shielding and geometric constraints, respectively.

\section{Optimisation of the SLN injection technique}

This study demonstrated a significant difference between nodal uptake from the two injection planes, with 10 times more nodal activity following intradermal than parenchymal plane injection. This concurs with McMasters et al, who reported a 5-to-7-fold increase of activity within nodes from intradermal compared to peritumoural injection [27].

Notwithstanding the small numbers of subjects in this study, we found that intradermal injection outperformed parenchymal injection and achieved a sensitivity of $100 \%$ for identification of disease within the axilla when IHC was used and when the false negative patient in whom wire localisation was performed prior to injection was excluded. This false negative case isconcerning, given the increasing practice of SLN identification after a negative ultrasoundguided axillary fine needle aspiration or loop wire insertion, and suggests that lymphatic flow may be disrupted by image guided intervention it is possible that in such patients tracer should 
be given by both intradermal and parenchymal injections. The possible effects of interventions on SLN tracer uptake and sentinel nodal identification merits further studies with larger series of patients.

Large nodes are no more likely to be involved with disease than small nodes. Thus, the largest nodes contained metastatic disease in only 39\% of patients. This increased to $50 \%$ when the 4 largest nodes in each patient were considered. Nodal sampling based on size alone, as advocated by some centres [28], cannot therefore be recommended.

\section{Resonances with Sappey's anatomical model}

The striking discordance of nodal echelon number identification by the two injection planes, with about one third of patients having near perfect correlation of uptake from the two injection sites, and one third having very poor correlation, may be explained by the variability in anatomical communication between the parenchymal and areolar lymphatics and the axillaas demonstrated by Sappey in 1874 from cadaveric mercury injections (figure 1a). For illustrative purposes, this image has been modified by the extension of the illustration to include axillary vessels and nodes (figure 1b). These images shows in beautiful detail the complexity of the network of minute lymphatics around the alveoli of the breast parenchyma that coalesce to form larger tracts. The lymphatic's around the areola (which form Sappey’s plexus) are larger in calibre The lymphatics from the parenchyma can either join those from the areola and drain to a common node (eg. node A, figure 1b), or may drain independently (eg node B, figure 1b) 
Close correlation between the two injection planes as seen in patients within group 1 may be explained by close proximity of the tumour and peritumoural injection to lymphatics which either coalesce towards the areola or become confluent with tracts which emanate from the areolar lymphatics, as would be the case as illustrated in Figure 1c. In contrast, discordant correlation from the two planes as seen in the patients of group 3, may be explained by location of a tumour and peritumoural injection close to parenchymal lymphatics which run independently to the axilla, discretely separated from areolar lymphatics, such as the case demonstrated in figure 1d. The relatively minute size of the tiny peripheral lymphatics compared to the larger lymphatic tracts which make up Sappey’s plexus may in part explain the high ratio of 10:1 in axillary tracer accumulation following periareolar injection compared to peritumoural injection. The absence, of any difference in this ratio across the three groups indicates that correlation between the two injection sites in terms of node echelon number does not depend on relative tracer uptakes at the two sites.

Ours is not the first study to use ex vivo well-counting to quantify tracer uptake within individual dissected lymph nodes following radiotracer breast injection. Two small studies used similar techniques, albeit with different study aims and tracers. Hultborn et al quantified axillary and parasternal nodal uptake of colloidal ${ }^{192}$ Gold injected at points as far distant as possible from breast tumours in patients about to undergo mastectomy and axillary clearance and internal mammary chain dissection [29]. They found that parenchymal injection resulted in nodal uptake within both the axilla and parasternal lymph node groups with an average uptake of 26-fold more activity in the axilla. They also noted that nodal disease impaired tracer uptake and resulted in unusual patterns of tracer distribution, echoing our finding of a negative trend between rank 
based on deposit size and rank based on tracer accumulation in patients with more than one diseased node.Pecking et al reported dual window lymph node counting and pathological correlation on an individual nodal basis, comparing nodal uptakes of ${ }^{111}$ In-labelled anticytokeratin monoclonal antibodies and ${ }^{99 \mathrm{~m}}$ Tc-labelled rhenium sulphide administered together subdermally in patients with breast cancer [30]. Uptake of antibody relative to colloid indicated a positive predictive value for antibody of $71 \%$ for the identification of nodal disease.

\section{Conclusion}

Using a dual isotope, well-counting technique that allows detailed quantification of lymphatic flow to individual nodes and correlation with pathology on a node-by-node basis, we report a novel approach designed to achieve a better understanding of the functional morphology of lymphatic drainage in the breast and to eradicate confounding factors that may have influenced previous studies. The technique illustrates functional differences in lymphatic drainage pathways from the intradermal and parenchymal planes that may be explained by anatomical differences in lymphatic arrangements from the breast as shown by Sappey's original model. Whilst this pilot

study may not have significantly advanced the clinical argument, it illustrates how the technique of well-counting of individual nodes has the potential to resolve this major controversy and justifies its application to larger series of patients.

\section{Acknowledgements}

We are very grateful to the Royal College of Radiologists of the United Kingdom for financial support. 


\section{Conflict of interest}

The authors state that they have no conflict of interest.

\section{References}

1. Deckers PJ. Axillary dissection in breast cancer: when, why, how much, and for how long? - another operation soon to be extinct? J Surg Oncol 1991;48:217-9.

2. Reintgen DS, Cruse CW, Wells K et al. The orderly progression of melanoma nodal metastases. Ann Surg 1994; 220:759-767

3. Statius Muller MG, Borgstein PH, Pijpers R et al. Reliability of the sentinel node procedure in melanoma patients: analysis of failures after long-term follow-up. Ann Surg Oncol 2000;7:461-8.

4. Tanis PJ, Nieweg OE, Valdes Olmos RA et al. Anatomy and physiology of lymphatic drainage of the breast from the perspective of sentinel node biopsy. J Am Coll Surg 2001; 192:399-409.

5. De Cicco C, Cremonesi M, Luini A et al. Lymphoscintigraphy and radioguided biopsy of the sentinel axillary node in breast cancer . J Nucl Med 1998;39:2080-4.

6. Linehan DC, Hill AD, Akhurst T et al. Intradermal radiocolloid and intraparenchymal blue dye injection optimise sentinel node identification in breast cancer patients. Ann Surg Oncol 1999;6:450-4. 7. Fleming FJ, Hill AD, Kavanagh D. Intradermal radioisotope injection optimises sentinel lymph node identification in breast cancer. Eur J Surg Oncol 2003; 29: 835-8.

8. De Cicco C, Trifiro G, Intra M. Optimised nuclear medicine method for tumour marking at sentinel node detection in occult primary breast lesions. Eur J Nucl Med Mol Imaging 2004;31:349-54. 9. Pelosi E, Baiocco A, Ala A et al. Lymphatic mapping in early stage breast cancer: comparison between periareolar and subdermal injection. Nucl Med Commun 2003;24:519-23. 
10. Pelosi E, Bello M, Giors M et al. Sentinel lymph node detection in patients with early-stage breast cancer: comparison of periareolar and subdermal/peritumoural injection techniques. J Nucl Med $2004 ; 45: 220-5$

11. Borgstein PJ, Meijer S, Pijpers RJ et al. Functional lymphatic anatomy for sentinel node biopsy in breast cancer: echoes from the past and the periareolar blue method. Ann Surg 2000;232:81-9.

12. Shimazu K, Tamaki Y, Taguchi T et al. Comparision between periareolar and peritumoural injection of radiotracer for sentinel lymph node biopsy in patients with breast cancer. Surgery 2002;131:277-86.

13. Reitsamer R, Peintinger F, Rettenbacher L. Subareolar subcutaneous injection of blue dye versus peritumoural injection of technetium-labeled human albumin to identify sentinel lymph nodes in breast cancer patients. World J Surg 2003;27:1291-4.

14. Roumen RM, Geuskens LM, Valkenburg JG. In search of the true sentinel node by different injection techniques in breast cancer patients. Eur J Sur Oncol 1999;25:347-51.

15. Maza S, Valencia R, Geworski L et al. Peritumoural versus subareolar administration of technetium-99m nanocolloid for sentinel lymph node detection in breast cancer: preliminary results of a prospective intra-individual comparative study. Eur J Nucl Med Mol Imaging 2003;30:651-6.

16. Vijayakumar V; Boerner PS; Jani A et al. A critical review of variables affecting the accuracy and false-negative rate of sentinel node biopsy procedures in early breast cancer Nucl Med Commun 2005;26:395-405.

17. Mariani G, Moresco L, Viale G et al. Radioguided sentinel lymph node biopsy in breast cancer. J Nucl Med 2001; 42:1198-1215. 
18. Mitterhauser M, Wadsak W, Key Mien L et al. The labelling of nanocoll with [ $\left.{ }^{111} \mathrm{In}\right]$ for dual isotope scanning. Appl Radiat Isot 2003;59:337-42.

19. Fowler JC, Solanki CK, Barber RW et al. Dual-isotope lymphoscintigraphy using albumin nanocolloid differentially labeled with ${ }^{111}$ In and ${ }^{99 m}$ Tc. Acta Oncol 2007;46:105-10.

20. Ergun EL, Ercan MT, Asansu A et al. Evaluation of ${ }^{99 m}$ Tc-HIG as a lymphoscintigraphic agent in rabbits. Nucl Med Commun 1998;19:665-70.

21. Svensson W, Glass DN, Bradley D et al Measurement of lymphatic function with Tc99m labeled polyclonal immunoglobulin. Eur J Nucl Med 1999;26:504-10.

22. Fowler JC, Solanki CK, Barber RW et al Measurement of the extraction fractions of nanocolloid and polyclonal immunoglobulin by axillary lymph nodes in patients with breast cancer. Nucl Med Commun 2004;25:935-940.

23. Pain SJ, Barber RW, Ballinger JR et al. Side-to-side symmetry of radioprotein transfer from tissue space to systemic vasculature following subcutaneous injection in normal subjects and patients with breast cancer. Eur J Nucl Med Mol Imaging 2003;30: 657-61.

24. Pain SJ, Barber RW, Ballinger JR et al. Transport of radiolabeled immunoglobulin injected into the web spaces of the hands of normal subjects and patients with breast cancerrelated lymphedema. J Vasc Res 2004;41:183-92.

25. Ellis IO, Pinder SE, Bobrow L. Guidelines for Pathology Reporting in Breast Cancer Screening NHS Breast Screening Programme Publications No 58; January 2005.

26. Turner RR, Ollila DW, Stern S et al. Optimal histopathologic examination of the sentinel lymph node for breast carcinoma staging. Am J Surg Pathol 1999;23:263-7.

27. McMasters KM, Wong SK, Martin RC et al. Dermal injection of radioactive colloid is superior to peritumoural injection for breast cancer sentinel node biops: results of a 
multiinstitutional study. Ann Surg 2001;233:676-87.

28. MacMillan RD, Barbera D, Hadjuminas DJ et al. Sentinel node biopsy for breast cancer may have little to offer four-node-samplers: results of a prospective comparison study. Eur J Cancer 2001;37:1076-80.

29. Hultborn KA, Larsson LG, Ragnhull I. The lymph drainage from the breast to the axillary and parasternal lymph nodes, studies with the aid of colloidal Au ${ }^{198}$ Acta Radiol 1955:43:52-64. 30. Pecking AP, Gougeon-Bertrand FJ, Lokiec FM et al. Radioimmunolymphoscintigraphy in the preoperative staging of primary breast cancer: A pilot study using a human monoclonal antibody. Int J Oncol 1996;9:659-67. 


\section{Legends}

Figure 1a Plate XIII of Anatomie, physiologie et pathologie des vaisseaux lymphatiques consideres chez l’homme et les vertebras by MPC Sappey. Publishers: A Delehaye \& E Lacrosnier, Paris, 1874. With thanks to the Royal Society of Medicine.

Figure 1b. An artist's supplementation of the Sappy illustration, extending the model to include lower axillary lymph vessels and nodes labelled A, B and C. Node A receives lymphatics which drain both the lower outer quadrant parenchyma and the areola. Node B receives lymphatics which drain only the upper outer quadrant parenchyma, and are independent of areolar lymphatic’s.

Figures 1c and 1d show intradermal periareolar and parenchymal peritumoural injections in yellow and red respectively. In both, the uptake of tracer into the finer parenchymal lymphatics from the peritumoural injection have resulted in less vessel and nodal uptake than uptake into the more substantial periareolar lymphatics following intradermal injection.

Figure 1c Illustrates a patient from group 1. The tumour is situated close to local microlymphatics that converge with the great tracts running from Sappey's plexus to the axilla, resulting in the same first (node A) and second (node B) echelon nodes from both routes.

Figure 1d. Illustrates a patient from group 3, The tumour is positioned close to local microlymphatics that join to form larger lymphatic vessels which course directly to the axilla 
independently of the larger lymphatic vessels of Sappey's areolar plexus whichare accessed by areolar, intradermal injection. These independent lymphatic routes of drainage from the two injection sites result in different first echelon (SLN) from the two routes, the periareolar injection to node A, the peritumoural injection site to node B. Both patients have a common second echelon node (C). 


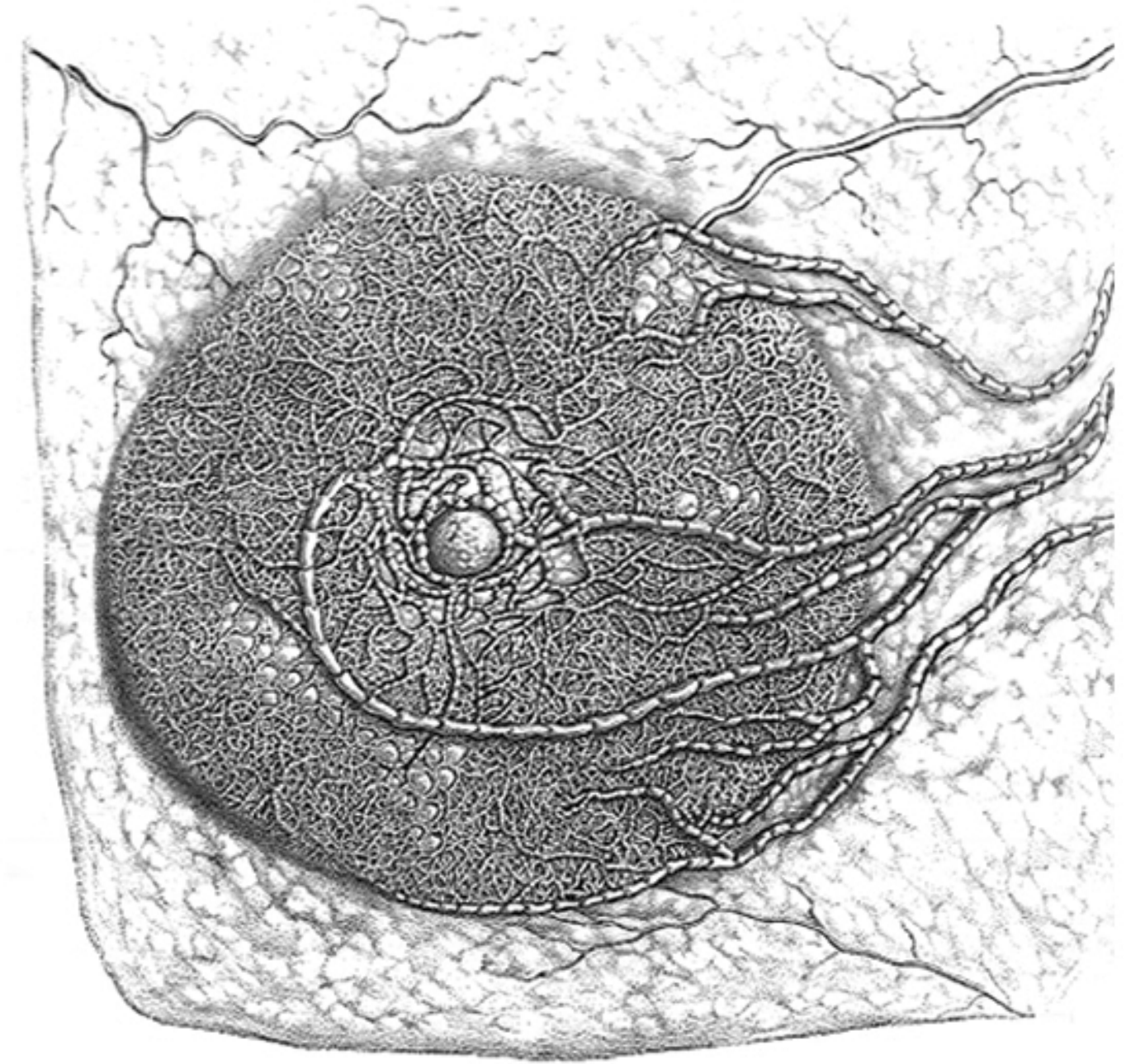


